\title{
Micro-Polymerase Chain Reaction Chip Reaction Chamber Temperature Measurement
}

\author{
Chan-Young Park, ${ }^{1,2}$ Seung-Cheol Lee, ${ }^{1,2}$ Yu-Seop Kim, ${ }^{1,2}$ \\ Hye-Jeong Song, ${ }^{1,2}$ and Jong-Dae Kim ${ }^{1,2^{*}}$ \\ ${ }^{1}$ Department of Convergence Software, Hallym University, \\ 1 Hallymdaehak-gil, Chuncheon-si, Gangwon-do 24252, Korea \\ ${ }^{2}$ Bio-IT Research Center, Hallym University, Hallym University, \\ 1 Hallimdaehak-gil, Chuncheon-si, Gangwon-do 24252, Korea
}

(Received March 1, 2015; accepted January 14, 2016)

Keywords: microfluidic channel, internal temperature, polymerase chain reaction, NTC-thermistor, thermocouple

In this paper, we discuss the correlation between the reagent temperature and the thermistor sensor outside the chamber. The polymerase chain reaction (PCR) chip employed is constructed on a printed circuit board (PCB) with a heating pattern and a thermistor at the opposite side of the chamber. Since the reaction chamber is made with various films and tapes, a thermocouple could be embedded easily to measure the reagent temperature within the chamber. The parameters for chip control, such as the maximum heating power and the temperature compensation coefficient, were determined using the measured temperatures. The results show that the parameters determined enabled the exact control of the reagent temperature during the polymerase chain reaction.

\section{Introduction}

Polymerase chain reaction (PCR) is an advantageous and critical technique required in modernday biological research because only a small amount of DNA is needed to run a test, and it can

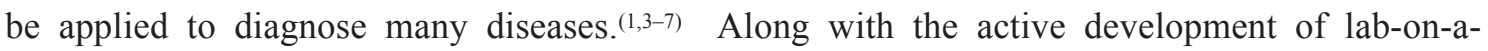
chip systems, which are analytical systems that overcome the disadvantages of existing genetic diagnostic techniques and allow on-site genetic diagnoses, micro-PCR chips containing microfluidic channels are also being developed. ${ }^{(1,2)}$

A micro-PCR chip can quickly determine various pieces of genetic information from a very small amount of DNA, enabling genetic analyses and diagnosis of various diseases in a very short time. However, the results of a PCR test rely greatly on the reaction temperature; hence it is necessary to control the temperature of the reaction as precisely as possible to ensure accurate results with reliable reproducibility and sensitivity. Therefore, the heater and temperature sensors need to be installed as close as possible to the chamber to control the temperature, and the temperature sensor may need to be calibrated before use. ${ }^{(2)}$

Temperature control of the micro-PCR chip employed in this study was achieved using a printed circuit board (PCB) pattern for heating, a fan for cooling, and a negative-temperature-coefficient (NTC) thermistor for temperature measurement. The thermistor was installed at the bottom of the PCB

*Corresponding author: e-mail: kimjd@hallym.ac.kr 
substrate, and it was necessary to confirm whether the temperature of the sample inside the chamber was actually the same as that of the thermistor. In this study, we installed a thermocouple inside the chamber of the micro-PCR chip to determine the reagent temperature in the chamber. The temperature measured with the thermocouple was compared with that of the thermistor and used to determine control parameters such as the maximum heating power and the amount of temperature compensation.

In $\S 2$, the details of the chip structure, the driving system, the thermocouple embedding method, and the power control and temperature compensation are illustrated. The experimental results and the conclusion are given in $\S \S 3$ and 4 , respectively.

\section{Materials and Methods}

The schematic of the micro-PCR chip used in this research is shown in Fig. 1. A black PCB was used as the substrate of the chip [left in Fig. 2(a)]. It had a heating pattern and soldering lands for a NTC thermistor that measured the temperature with a resistance tolerance of $0.5 \%$ (NCP15XH103D03RC, Murata Manufacturing Co., Ltd.) at the bottom. The top of the substrate included a rectangular copper plate for thermal spreading, at the upper side of which white silk screen printing ink was printed for fluorescence scattering [right in Fig. 2(a)]. A box tape with a thickness of $50 \mu \mathrm{m}$ (\#309 Mini Clear, 3M Co., Ltd.) was attached on the top of the black PCB substrate to prevent the biological molecules from adsorbing onto the PCB (Fig. 1). A black double-sided tape of $400 \mu \mathrm{m}$ thickness (No. 5620BWN, Nitto Denko Co., Ltd.), from where the
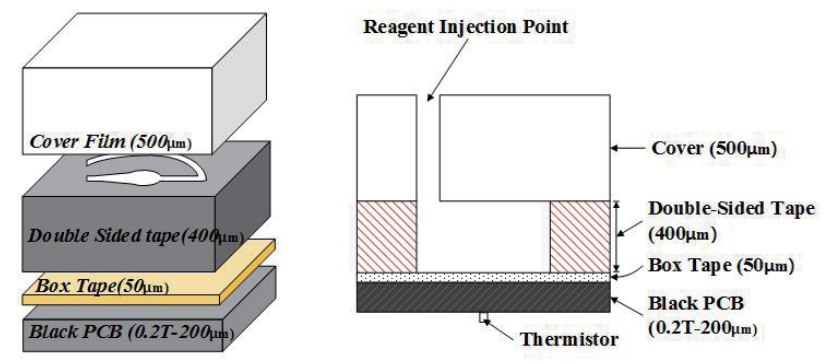

Fig. 1. (Color online) Lateral schematic view of the micro-PCR chip.

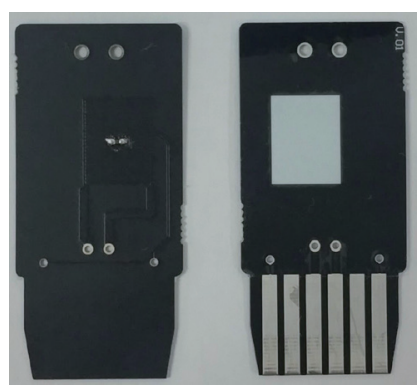

(a)

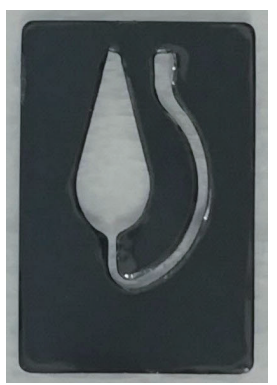

(b)

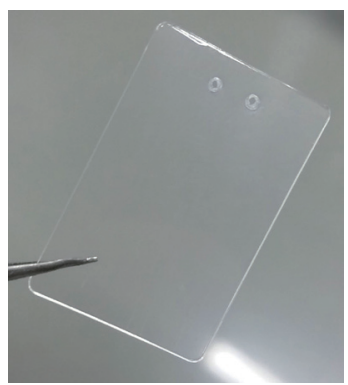

(c)

Fig. 2. Materials used to construct the micro-PCR chip. (a) Back (left) and front (right) of the black PCB. (b) Double-sided tape. (c) PP cover film. 
chamber shape was cut out [Fig. 2(b)], was attached over the box tape (Fig. 1). The double-sided tape preserved its properties and endured expansion due to high temperatures up to $95{ }^{\circ} \mathrm{C}$ according to the data provided from the company. A polypropylene (PP) cover film of $500 \mu \mathrm{m}$ thickness with two holes for injecting and retrieving the sample [Fig. 2(c)] was attached on top of the double-sided tape (Fig. 1).

The driving system of the PCR chip was in a host-local architecture, where the host system was a computer with Microsoft Windows operating system. The PIC18F4553 (Microchip Technology Inc.) embedded in the local system measured the temperature and sent the value to the host. The host determined the control parameters, such as the duty of the pulse width modulation (PWM) for heating or the fan on/off for cooling, on the basis of the value received.

This host-local system with the micro-PCR chip employed in this research was primarily developed to detect fluorescence through the transparent PP cover during a PCR test using an optic module with a LED, a photodiode, and the corresponding optics. However, a USB microscope was used in this research instead of the optical module to observe the internal changes of the chamber due to temperature changes (Fig. 3).

A K-type thermocouple of $50 \mu \mathrm{m}$ diameter without a sheath (CHAL-002, Omega Co., Ltd.) was integrated vertically within the micro-PCR chip chamber to measure the actual temperature of the reagent as shown in Fig. 4. The vertical alignment of the thermocouple prevented interference due to air bubbles that rose at high temperatures. The air bubbles could induce noise or decrease the temperature measured if they stuck on the thermocouple wire bonding point. This alignment minimized such incidents as observed using the USB microscope. The temperature of the thermocouple was measured with an eight-channel thermocouple reader with $\pm 0.5{ }^{\circ} \mathrm{C}$ accuracy and 100 ms conversion time (TC-08, Omega Co., Ltd.).

Since the micro-PCR host system used in this research was programmed with the Microsoft Foundation Class (MFC) library, the thermocouple temperature sampling function was easily integrated using the Windows library provided by the manufacturer. By using the integrated application, the thermistor and the thermocouple temperatures could be concurrently measured. The measurements were performed over only 6 cycles instead of the full PCR cycles because they were sufficient for the statistical analysis of the temperature.

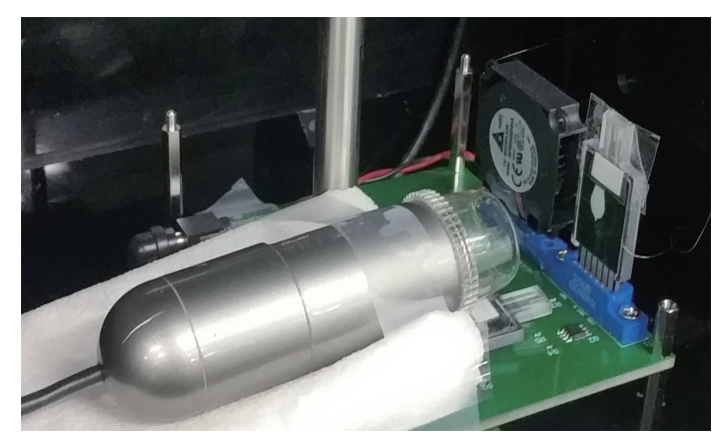

Fig. 3. (Color online) Experimental setup.

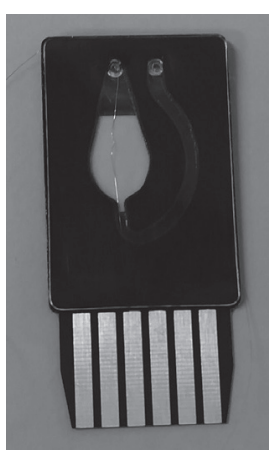

(a)

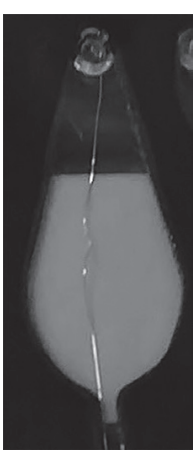

(b)
Fig. 4. Thermocouple attached to the micro PCR chip (a) and an enlarged picture of the chamber (b). 
In this study, two kinds of control parameters were investigated on the basis of the temperature measured. First, the heating power must be controlled with precision to secure PID control and prevent local overheating. In this experiment, various heating powers were implemented by turning on the PWM device in the embedded controller during 1,2,3, and $4 \mathrm{~ms}$ intervals of the period of $4 \mathrm{~ms}$, which correspond to one-quarter, half, three-quarters, and full power, respectively. The behavior of the thermocouple temperature, which represented that of the reagent, was analyzed, and the best heating power was determined.

Second, the temperature of the reagent was estimated from the relationship between the temperatures of the thermistor and the thermocouple. The results showed that the thermocouple and thermistor temperatures had a very linear relationship, where the slope was slightly less than unity and the intersection was approximately zero. Therefore, the reagent temperature could be estimated by multiplying the thermistor temperature by the slope.

\section{Results}

The heating power was controlled by activating the PWM device for 1, 2, 3, and $4 \mathrm{~ms}$ intervals of the period of $4 \mathrm{~ms}$. The heating temperature was controlled following the common PID method with PWM. Figure 5 shows the temperature change of the thermistor (solid line) and thermocouple (dotted line). The cooling interval $\left(95 \rightarrow 60{ }^{\circ} \mathrm{C}\right.$ ) was not affected by the amount of power since the system only turned on the fan to cool down. The temperature ramping speed for heating was calculated using the temperature difference and the rise time from 10 to $90 \%$ of the step temperature between the start and the target temperature. However, the temperature ramping speed over the interval $25 \rightarrow 95{ }^{\circ} \mathrm{C}$, which is actually the interval where the temperature increases from room temperature to $95{ }^{\circ} \mathrm{C}$, was calculated using the temperature and time difference between 40 and $90{ }^{\circ} \mathrm{C}$ to eliminate the ambiguity of the term room temperature. The starting point to measure the ramping speed is marked with " $\Delta$ " or " $\nabla$ " for heating or cooling, respectively, and the end point is marked with "O" in Fig. 5.

The ramping speed for each interval according to heating power is shown in Table 1, and the bottommost rows show the mean, standard deviation (STD), coefficient of variation (CV), and slope of the ramping speed.

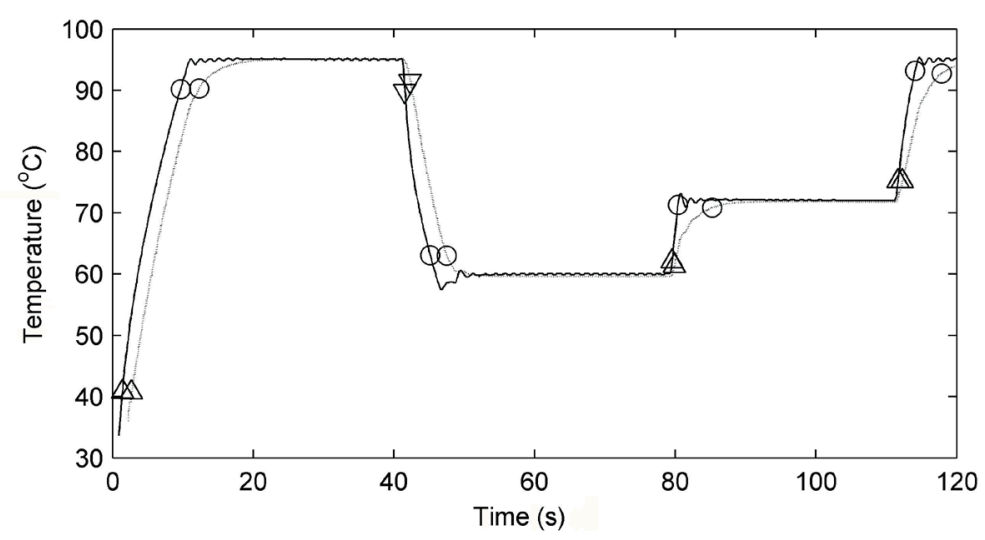

Fig. 5. Temperature change of thermistor (solid line) and thermocouple (dotted line) during one cycle of PCR. The ramping speed was calculated as the temperature change per second from " $\Delta$ " or " $\nabla$ " to " $\circ$ ". 
Table 1

Temperature ramping speed of thermistor and thermocouple according to the heating power $\left({ }^{\circ} \mathrm{C} / \mathrm{s}\right)$.

\begin{tabular}{|c|c|c|c|c|c|c|c|c|}
\hline & \multicolumn{4}{|c|}{ Thermistor } & \multicolumn{4}{|c|}{ Thermocouple } \\
\hline Power & $25 \rightarrow 95$ & $95 \rightarrow 60$ & $60 \rightarrow 72$ & $72 \rightarrow 95$ & $25 \rightarrow 95$ & $95 \rightarrow 60$ & $60 \rightarrow 72$ & $72 \rightarrow 95$ \\
\hline $1 / 4$ & 5.97 & -7.42 & 11.50 & 7.55 & 5.17 & -5.47 & 1.77 & 3.14 \\
\hline $2 / 4$ & 16.35 & -7.04 & 15.50 & 18.30 & 7.18 & -5.22 & 1.52 & 2.91 \\
\hline $3 / 4$ & 30.94 & -7.74 & 23.19 & 26.99 & 6.92 & -5.33 & 1.45 & 2.88 \\
\hline 1 & 41.27 & -7.41 & 27.87 & 37.80 & 7.46 & -5.38 & 1.33 & 2.91 \\
\hline Mean & 23.63 & -7.41 & 19.52 & 22.66 & 6.68 & -5.35 & 1.52 & 2.96 \\
\hline STD & 15.59 & 0.29 & 7.39 & 12.85 & 1.03 & 0.10 & 0.19 & 0.12 \\
\hline CV (\%) & 65.99 & -3.88 & 37.85 & 56.69 & 15.43 & -1.90 & 12.20 & 4.06 \\
\hline Slope & 12.05 & -0.07 & 5.68 & 9.94 & 0.66 & -0.02 & -0.14 & -0.07 \\
\hline
\end{tabular}

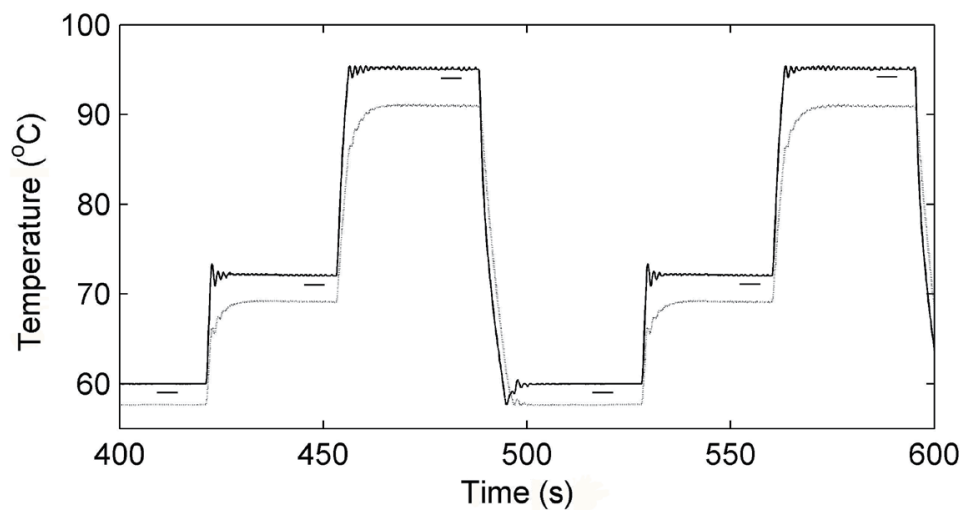

Fig. 6. Thermistor (solid line) and thermocouple (dotted line) temperature profile. The line segments below the thermistor temperature profile indicate the intervals for averaging.

As shown in Table 1, the average cooling speed of the thermistor and thermocouple were 7.41 and $5.35{ }^{\circ} \mathrm{C} / \mathrm{s}$, respectively. The $\mathrm{CV}$ of these values was less than $3.88 \%$ because it was not affected by the heating power since the system only uses a fan for cooling.

The heating slopes of the thermistor given in the last row of Table 1 show that the temperature increased rapidly in proportion to increased power. However, the slopes of the line for the thermocouple did not increase with increasing power, and the speed change was less than 0.1 for the intervals $60 \rightarrow 72{ }^{\circ} \mathrm{C}$ and $72 \rightarrow 95^{\circ} \mathrm{C}$, which were critical in the PCR cycle. This indicated that $1 / 4$ power was the best choice for conducting the experiments in this study.

Figure 6 shows the temperature change for one cycle. The temperature was measured every 50 ms. Table 2 shows the mean and STD of the average temperature at 95,60 , and $72{ }^{\circ} \mathrm{C}$ measured using the thermistor and thermocouple. The temperature values used for the calculation were read once the target temperature was stabilized, and the stabilized interval is indicated with a line beneath the thermistor profile graph in Fig. 6. The average temperature was calculated for 12 stabilized intervals at $95^{\circ} \mathrm{C}$, and 11 intervals for both 60 and $72{ }^{\circ} \mathrm{C}$. As can be seen in the table, the thermistor temperature showed the exact same temperature as the target temperature and had low STD. This resulted from the chip driving system controlling the PID according to the temperature measured by the thermistor. However, the temperature measured by the thermocouple showed temperatures lowered by $-4.1,-2.3$, and $-2.8^{\circ} \mathrm{C}$ for 95,60 , and $72{ }^{\circ} \mathrm{C}$, respectively. Therefore, the 
Table 2

Averaged temperatures of thermistor and thermocouple.

\begin{tabular}{lcccc}
\hline & Target temperature & 95 & 60 & 72 \\
\hline \multirow{2}{*}{ Thermistor } & Mean & 95.0 & 60.0 & 72.1 \\
\cline { 2 - 5 } & STD & 0.01 & 0.00 & 0.05 \\
\hline \multirow{2}{*}{ Thermocouple } & Mean & 91.0 & 57.7 & 69.2 \\
\cline { 2 - 5 } & STD & 0.19 & 0.04 & 0.37 \\
\hline Mean error & & -4.1 & -2.3 & -2.8 \\
\hline
\end{tabular}

Table 3

Averaged temperatures of thermistor and thermocouple after correction.

\begin{tabular}{lcccc}
\hline \multirow{2}{*}{ Thermistor } & Target temperature & 95 & 60 & 72 \\
\cline { 2 - 5 } & Mean & 95.0 & 60.0 & 72.0 \\
\hline \multirow{2}{*}{ Thermocouple } & STD & 0.01 & 0.01 & 0.00 \\
\cline { 2 - 5 } & Mean & 95.2 & 60.3 & 72.2 \\
\hline Mean error & STD & 0.05 & 0.01 & 0.02 \\
\hline
\end{tabular}

temperature measured by the thermistor should be corrected to represent the reagent temperature. Fortunately, since the relationship between the thermistor and the thermocouple temperatures was very linear with the intersection value close to zero, the reagent temperature could be easily corrected by multiplying the thermistor temperature by the slope of the linear relationship.

Table 3 shows the mean and STD of the thermistor and thermocouple temperatures after correction. The mean temperature difference decreased to less than $0.3^{\circ} \mathrm{C}$ after correction.

\section{Conclusions}

In this study, we examined the correlation between the thermocouple temperature inside the reagent chamber and that of the thermistor on the opposite side of the chamber. The measured reagent temperature was used to determine the control parameters such as the heating power and the correction coefficient during the reaction.

Only one-fourth of the maximum heating power was sufficient when controlling the increase in the temperature because the temperature increase time of the reagent was not affected even if more power was used.

As the temperatures of the thermocouple and the thermistor were highly linear with zero interception, simply multiplying the thermistor temperature by a constant less than unity gave the thermocouple temperature. The thermocouple temperature, which represents that of the reagent, reached the target temperature accurately with this simple correction.

The proposed scheme can be applied to similar kinds of PCR chips owing to the availability of very thin thermocouple wires. The temperature dynamics of other PCR chips should be identified because they tend to change according to the reagent mixtures and chamber shape; the thermal capacity of the reagents cannot be ignored owing to the small size of the chips. 


\section{Acknowledgements}

This work was supported by Hallym University Research Fund, 2015 (HRF-201510-015) and

Basic Science Research Program through the National Research Foundation of Korea (NRF) funded by the Ministry of Education (2013R1A1A2013490).

\section{References}

1 C. Koo, M. Malapi-Wight, H. S. Kim, O. S. Cifci, V. L. Vaughn-Diaz, B. Ma, S. Kim, H. Abdel-Raziq, K. Ong, Y.-K. Jo, D. C. Gross, W.-B. Shim, and A. Han: PloS One 8 (2013) e82704.

2 E. Salm, Y.-S. Liu, D. Marchwiany, D. Morisette, Y. He, L. Razouk, A. K. Bhunia, and R. Bashir: Biomed. Microdevices 13 (2011) 973.

3 J. J. Ramsden: Biomedical Surfaces (Artech House, Norwood, MA, 2007).

4 M. Malmsten: Biopolymers at Interfaces (Taylor \& Francis, New York, 2003).

5 C. Zhang and D. Xing: Nucleic Acids Res. 35 (2007) 4223.

6 C. Zhang, D. Xing, and Y. Li: Biotechnol. Adv. 25 (2007) 483.

7 D. P. Herzog: IVD Technology (2006).

8 R. Kodziusa, K. Xiaoc, J. Wud, X. Yid, X. Gongd, I. G. Fouldsb, and W. Wen: Sens. Actuators, B 161 (2012) 349.

9 C. Plaisant, A. Rose, B. Shneiderman, and A. J. Vanniamparampil: Software IEEE 14 (1997) 66.

10 J. D. Kim, Y. U. Lee, and S. Kim: IEICE Trans. 86 (2003) 859.

11 J. D. Kim, J. Kim, and G. Lee: Proc. SPIE 7929 (2011) 79290Y-79290Y-9.

12 C. Y. Park, J. D. Kim, J. H. Ku, Y. S. Kim, H. J. Song, and J. W. Kim: Sens. Lett. 10 (2012) 1197. 